\title{
ANÁLISE DAS DIFICULDADES RELACIONADAS ÀS ATIVIDADES DIÁRIAS DE CRIANÇAS E ADOLESCENTE COM DIABETES MELLITUS TIPO 1: DEPOIMENTO DE MÃES
}

\author{
Maria Lúcia Zanetti ${ }^{1}$ \\ Isabel Amélia Costa Mendes ${ }^{2}$
}

Zanetti ML, Mendes IAC. Análise das dificuldades relacionadas às atividades diárias de crianças e adolescente com diabetes mellitus tipo 1: depoimento de mães. Rev Latino-am Enfermagem 2001 novembro-dezembro; 9(6):25-30.

Estudo descritivo com o objetivo de analisar as dificuldades que as mães de crianças e adolescentes diabéticos tipo 1 apresentam face as atividades diárias com o filho, partindo da premissa de que fatores de ordem econômica, social, pessoal e emocional da família interferem para obtenção de um bom controle metabólico. Através de roteiro semi-estruturado de entrevista aplicado a trinta mães, obteve-se os dados cuja análise obedeceu às normas preconizadas para estudos descritivos. Os resultados permitiram concluir que as mães apontaram como dificuldades nas atividades diárias com os filhos: reuniões de convivência social; motivá-lo a realizar atividade física; dieta; adaptação escolar; relacionamento com a equipe de saúde e comportamento do filho frente aos demais irmãos. Foi evidenciada a necessidade de trabalho planejado e integrado por equipe multiprofissional junto às famílias, direcionado para cada dificuldade abordada, observando-se sua interdependência para o êxito do cuidado à criança e adolescente diabético tipo 1.

DESCRITORES: criança, adolescência, diabetes mellitus tipo 1

\section{ANALYSIS OF DIFFICULTIES REGARDING THE DAILY ACTIVITIES OF TEENAGERS AND CHILDREN WITH TYPE-1 DIABETES MELLITUS: MOTHERS' ACCOUNTS}

This descriptive study aimed at analyzing the difficulties faced by mothers of type-1 diabetic teenagers and children in view of the child's daily activities and based on the principle that emotional, social, personal and economic factors concerning the family interfere with achieving good metabolic control. Data were obtained by means of a semi-structured interview guide applied to 30 mothers. Data analysis followed the standards preconized for descriptive studies in which directed interviews are used. Results showed that: mothers have difficulties in dealing with children's daily activities such as social gatherings, in motivating them to develop physical activities, diet, school adjustment, relationship with the health team as well as with the children's behaviors regarding their brothers/sisters. Results indicated the need for planned and integrated work by a multiprofissional team with the families, considering all the aspects addressed in this study and their interdependence, aiming at a successful care to diabetic children and adolescents.

KEY WORDS: child, adolescence, type-1 diabetes mellitus

\section{ANÁLISIS DE LAS DIFICULTADES RELACIONADAS CON LAS ACTIVIDADES DIARIAS DE LOS NIÑOS Y ADOLESCENTES CON DIABETES MELLITUS TIPO 1: DECLARACIONES DE LAS MADRES}

Estudio descriptivo con el objetivo de analizar las dificultades que las madres de los niños y adolescentes diabéticos tipo 1, presentan de cara a las actividades diarias con el hijo; partiendo de la premisa de que factores de orden económico, social, personal y emocional de la familia interfieren para la obtención de un buen control metabólico. A través de un guión de entrevista semi-estructurada aplicado a treinta madres, se obtuvieron los datos, cuyo análisis se realizó según las normas preconizadas para estudios descriptivos. Los resultados permitieron concluir que las madres anotan como dificultades en las actividades diarias con los hijos: reuniones de convivencia social, motivarlo a realizar la actividad física, dieta, adaptación escolar, relación con el equipo de salud y el comportamiento del hijo frente a los demás hermanos. Se hizo evidente la necesidad de un trabajo planeado e integrado por un equipo multiprofesional al lado de las familias, orientado hacia cada dificultad anotada, teniendo en cuenta su interdependencia para el éxito del cuidado del niño y del adolescente diabético tipo 1.

DESCRIPTORES: niño, adolescencia, diabetes mellitus tipo 1

${ }^{1}$ Enfermeiro, Professor Doutor da Escola de Enfermagem de Ribeirão Preto da Universidade de São Paulo, Centro Colaborador da OMS para o desenvolvimento da pesquisa em enfermagem, e-mail: zanetti@eerp.usp.br; ${ }^{2}$ Enfermeiro, Professor Titular da Escola de Enfermagem de Ribeirão Preto da Universidade de São Paulo, Centro Colaborador da OMS para o desenvolvimento da pesquisa em enfermagem 


\section{INTRODUÇÃO}

Atualmente o aumento do número de pessoas com doenças crônico-degenerativas tem se constituído em um desafio para os serviços de saúde e para a sociedade. Segundo a Organização Mundial da Saúde, o diabetes mellitus tipo 1 é uma das mais importantes doenças crônicas da infância em esfera mundial. Nos Estados Unidos da América dos 651000 casos novos diagnosticados a cada ano, 11000 são em crianças e adolescentes, constituindo-se assim na segunda mais importante doença crônica infantil neste país ${ }^{(1)}$.

Mesmo considerando a disponibilidade de terapêuticas efetivas, é ainda preciso compreender o quão penoso torna-se 0 tratamento para os portadores, famílias e sociedade, pois o diabetes tipo 1 freqüentemente progride com seqüelas, tais como: amputação, cegueira, nefropatia e retinopatia, comprometendo a qualidade de vida da pessoa.

Em particular, o diabetes mellitus requer da criança e adolescente diabético, das famílias e dos profissionais de saúde, esforços conjuntos para que os portadores atinjam um bom controle metabólico, a fim de minimizar as complicações advindas a longo prazo.

Estes esforços devem ser direcionados para ajudar a criança e 0 adolescente a administrar o complexo regime de insulina, dieta e exercícios a fim de manter os níveis de glicose sangüínea dentro dos limites de normalidade, proporcionando-lhes qualidade de vida.

O impacto de uma doença crônica, no caso o diabetes mellitus, sobre a família pode ocorrer nas esferas comportamental, somática, social e financeira, sendo freqüente encontrá-las interrelacionadas ${ }^{(2)}$.

No entanto, a maior parte da literatura sobre o impacto da doença crônica na infância nas famílias têm dado ênfase à figura materna na relação com filho doente. Isto justifica-se, pelo fato de que as mães estão mais envolvidas no cuidado diário dos filhos, 0 que as torna sensíveis à doença e aos seus efeitos, levando-as a perceber as conseqüências sobre os outros membros da família. Outras razões também são apontadas: em geral são as mães que levam os filhos ao consultório médico, fazem observações e participam dos grupos de pais, e portanto constituem, elas mesmas, uma rica fonte de dados.

Famílias estruturadas e organizadas podem fornecer um ambiente mais compatível para as necessidades da criança e adolescente diabético, pois o próprio tratamento exige controle e organização. Os sentimentos como superproteção, indiferença ou descuido com crianças e adolescentes diabéticos podem contribuir para a obtenção de um mau controle metabólico. Por outro lado, os pais que levam as crianças e adolescentes a assumirem toda a responsabilidade pelo auto-cuidado, também têm demonstrado dificuldades para manter os níveis glicêmicos compatíveis com 0 controle metabólico ${ }^{(3)}$.

Nesta direção, a relação entre auto-cuidado e controle metabólico em crianças e adolescentes diabéticos tipo 1 exige algum grau de envolvimento dos pais no cuidado. As crianças e adolescentes que demonstraram maior independência para as atividades de autocuidado não tiveram necessariamente o melhor controle metabólico em relação àquelas cujos pais estiveram mais envolvidos no cuidado diário em relação ao diabetes ${ }^{(4)}$.

Parece-nos que o equilíbrio entre independência e dependência da criança doente necessita ser abordado dentro do contexto familiar. Para administrar os cuidados que uma doença crônica exige, tal como o diabetes em crianças e adolescentes, necessita-se de uma abordagem para o cuidado, através de uma equipe multiprofissional tendo a criança, 0 adolescente e a família como foco central deste cuidado ${ }^{(5)}$

Tal abordagem justifica-se pois os pais enfrentam uma diversidade de problemas em relação ao diabetes mellitus tipo 1 , sendo que os mais freqüentes estão relacionados à administração de insulina, dieta, dinâmica familiar e testes de glicose no sangue $e$ urina ${ }^{(6)}$.

Considerando ainda a diversidade de problemas enfrentados pelos pais, o diabetes em criança pode ter efeito profundo na dinâmica familiar, situação esta observada quando os pais assumem a administração de injeções de insulina e as análises de sangue, pois, estes ficam em conflito e acabam não tendo outra opção a não ser magoar o filho ${ }^{(7)}$. Problemas conjugais e outras dificuldades familiares poderão resultar da não aceitação do diagnóstico, comprometendo o controle do diabetes na criança ${ }^{(2)}$. Em relação aos irmãos, estes poderão ter medo de vir a desenvolver a doença, como também, sentir ciúmes da atenção dispensada ao irmão recémdiagnosticado.

Atentas a estas questões, passamos a nos interessar pelo estudo da problemática que cerca as mães de crianças e adolescentes portadores de diabetes mellitus tipo 1, com a finalidade de avaliar 0 impacto desta doença para os pacientes e a sua família. 0 objetivo proposto é o de analisar as dificuldades que as mães de crianças e adolescentes diabéticos tipo 1 apresentam, face às atividades diárias com o filho portador de diabetes.

\section{MÉTODOS}

Trata-se de um estudo descritivo e seu desenho atende aos pré-requisitos de um estudo de caso. A população foi constituída por 30 (trinta) mães de crianças e adolescentes diabéticos tipo 1 de escolas de $1^{\circ} \mathrm{e} 2^{\circ} \mathrm{grau}$, públicas e particulares de Ribeirão Preto-SP. 
O instrumento utilizado para a coleta de dados foi um roteiro semi-estruturado para entrevista, considerando as variáveis relacionadas às atividades diárias com o filho portador de diabetes (alimentação, atividades escolares, lazer, exercício físico e relacionamento familiar), contendo quinze questões semi-abertas. As mães foram localizadas nas escolas, por meio da aplicação de um formulário visando a identificação de crianças e adolescentes portadores de diabetes. Em fase subseqüente, dirigimo-nos às residências das famílias esclarecendo às mães o objetivo e a natureza da investigação, quando foi obtido o consentimento verbal para a coleta de dados assegurando o anonimato e o sigilo das respostas.

A coleta de dados foi realizada na residência da família, em ambiente privado onde 0 entrevistado não tivesse muitas interferências, garantindo-se desse modo tranqüilidade na emissão das respostas. As entrevistas tiveram duração média de sessenta minutos. As respostas às questões semi-abertas foram registradas no próprio instrumento, concomitantemente à entrevista.

Para análise dos dados, às variáveis relacionadas as atividades diárias com o filho portador de diabetes foram atribuídos códigos específicos. As questões assim codificadas foram transpostas para uma planilha. A estrutura do banco de dados foi confeccionada a partir das planilhas sendo formatado no programa FOX PRO-2. A análise obedeceu às normas preconizadas para um estudo descritivo.

\section{RESULTADOS E DISCUSSÃO}

Ao investigar as dificuldades que as $30(100 \%)$ mães têm para ajudar o filho nas atividades diárias para o controle do diabetes, encontramos uma lista com nove ítens, quais sejam: dieta, administração de insulina, financeiras, emocionais, viagens de férias, proteção excessiva, festas e reuniões familiares, insegurança e atividades escolares. Das nove dificuldades apresentadas 14 (46,7\%) mães associaram três dificuldades, $8(26,7 \%)$ mães associaram quatro dificuldades, e $5(16,7 \%)$ mães associaram cinco dificuldades (Figura 1).

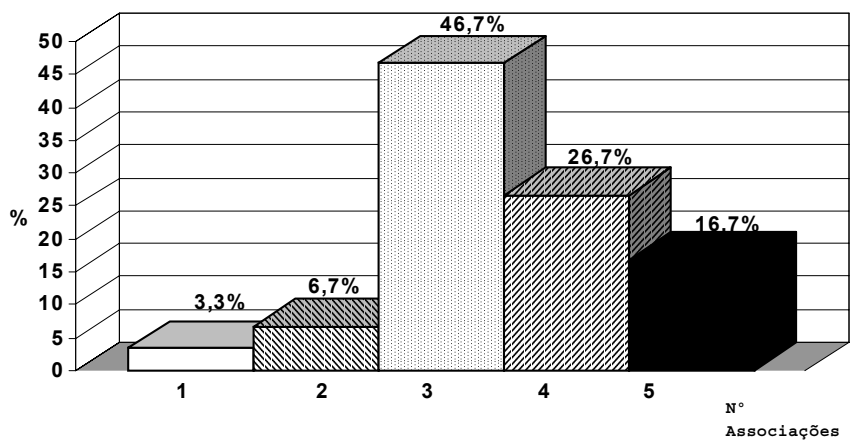

Figura 1 - Distribuição das mães de crianças e adolescentes com diabetes mellitus tipo 1 , matriculados em escolas de $1^{\circ}$ e $2^{\circ} \mathrm{grau}$, segundo o número de associações de dificuldades para ajudar o filho no controle do diabetes, Ribeirão Preto-SP, 1997
As associações mais freqüentes estão relacionadas à dieta, atividades escolares, atividades físicas, comportamentos dos filhos frente aos demais membros da família e relacionamento com a equipe de saúde. Em relação as atividades diárias com os filhos diabéticos, todas as mães referiram que precisaram de algum tempo para reorganizá-las e, $28(93,3 \%)$ relataram que têm um controle de horários e exigências diferentes na rotina diária de suas atividades para com o filho doente em relação aos demais.

Dentre as dificuldades que as mães de crianças e adolescentes com diabetes mellitus tipo 1 relataram, a dieta constituiuse em uma das principais, apontando que todas as mães tiveram que reestruturar o cardápio alimentar da família sendo em 26 (86,7\%) as dificuldades foram em relação à convivência social, conforme podemos constatar pelos depoimentos abaixo mencionados:

... Tive que restringir a compra de doces, refrigerantes e massas, isso trouxe um transtorno para os irmãos. ... Não aceito convites para festas ... A minha filha é louca por doces e chocolates ...

... a vigília é constante, a informação que o diabetes está controlado é falso. Não passa um dia sem alteração. No começo foi horrivel, muito difícil. O médico colocou em quatro folhas de papel as orientações sobre a alimentação e não explicou nada, fiquei apavorada. Os alimentos prescritos eram extremamente caros, difíceis de serem encontrados e não fazia parte da nossa alimentação habitual. Depois fui entendendo que poderia ser diferente e fiz as adaptações necessárias. Deixei de participar de reuniões festivas junto com o filho...

Atualmente, recomendações dietéticas estão baseadas em princípios de uma alimentação saudável e bons hábitos alimentares, indicadas para toda a família. Uma dieta baixa em açúcar e gordura e rica em alimentos com alto teor de carboidratos e fibras é recomendada em refeições regulares ${ }^{(8-9)}$.

Mesmo reconhecendo que as recomendações atuais ajudam a tornar menos penoso a dieta alimentar para crianças e adolescentes com diabetes mellitus tipo 1 , as taxas de não aderência à dieta em crianças em idade escolar é da ordem de 40 a $80 \%{ }^{(10)}$. Esta também parece ser uma das maiores dificuldades encontradas pelas mães, no nosso estudo. Alguns fatores têm sido associados com uma baixa aderência em relação à dieta, tais como: participação em eventos sociais, passeios em restaurantes, viagens de férias, companhia dos amigos e alterações emocionais.

Aderir a um plano alimentar envolve mudanças apropriadas que se iniciam dentro da própria família e o êxito deste processo exige mecanismos de adaptação para promover tais mudanças. Parece que a maior dificuldade relaciona-se com os carboidratos de absorção rápida tais como: doces, balas e chocolates, pois a freqüência relatada pelas mães, cujos filhos deixaram de participar de reuniões festivas é relevante.

Os depoimentos das mães indicam comprometimento dos hábitos alimentares em termos qualitativos, antes mesmo do filho 
tornar-se diabético. No entanto, os dados parecem indicar redirecionamento da qualidade dos alimentos consumidos pelo grupo familiar como um todo, após a confirmação do diagnóstico do filho. Entretanto, a reestruturação do cardápio traz alguns transtornos para os irmãos que se sentem afetados pelas mudanças nos hábitos alimentares.

Ainda neste tópico, verificamos que as mães precisam ser melhor informadas e orientadas no sentido de que a alimentação do filho deve ser fracionada em desjejum, colação, almoço, merenda, jantar e ceia, adequando assim as refeições ao tipo e número de aplicação diária de insulina e à atividade física a ser realizada.

Mais uma vez, parece-nos que mudar comportamentos em relação à dieta de criança e adolescente passa, obrigatoriamente, pela educação do grupo familiar. Outro aspecto que queremos aqui enfatizar aos profissionais de saúde é que valorizem e considerem as preferências alimentares, o significado do alimento para as famílias e as limitações sócio-econômicas para adquiri-los.

As dificuldades relacionadas às atividades escolares foram manifestadas por $9(30,0 \%)$ das mães, que referiram perceber prejuízos no processo educacional do filho, relatando sentirem-se também inseguras e com medo do filho apresentar episódios de hipoglicemia na escola, acreditando que os professores não estão preparados para socorrê-los nesta situação. Apresentamos a seguir os relatos das mães sobre esse tema:

... A minha filha transferiu várias vezes de escola, não queria freqüentar mais a escola, até que abandonou os estudos na $6^{2}$ série. Observei que a alfabetização foi prejudicada e as notas pioraram. Eu fazia reunião com os professores mas não sentia interesse dos mesmos em relação ao problema da minha filha. Eles queriam apenas o atestado médico para liberação da educação física ...

... A alfabetização da minha filha foi prejudicada. Tem dificuldade de aprender. 0 meu marido já fez até teste de quociente intelectual e o resultado foi normal. Os professores ajudam na supervisão do lanche, mas eles reclamam que ela dorme sentada na carteira. Tem problemas com os colegas de classe que dizem: ela tem sangue de água ...

... Os professores da escola de meu filho foram até ao grupo de apoio à criança diabética do hospital das clínicas para receber informações de como lidar com ele. Os elementos do grupo de apoio também visitavam a escola de meu filho ...

Parece ser necessário pensar no estabelecimento de uma via de comunicação entre os membros da equipe multiprofissional de saúde; por exemplo: o enfermeiro com os professores das escolas. Os pais ficam freqüentemente preocupados sobre como o professor do filho reconhecerá os sinais e sintomas da hipoglicemia para ajudar a socorrê-lo nestas ocasiões. Por outro lado, reconhece-se que os professores podem ter pouco ou nenhum conhecimento, ou experiência com diabetes ${ }^{(7)}$.

Os depoimentos das mães mostraram-nos que a problemática vivida pela criança e adolescente com diabetes mellitus tipo 1 pode afetar a alfabetização; no entanto, estudos precisam ser desenvolvidos para aprofundamento desta questão.

Em relação ao despreparo dos professores diante da possibilidade de atendimento de crianças em situações de hipoglicemia, visualizamos que a disponibilidade de cursos de primeiros socorros nas escolas poderia ajudá-los a lidar com estas emergências, aliviando sua tensão e medo, assim como dos seus pais ${ }^{(11)}$.

No que se refere às atividades físicas realizadas, os resultados apontam que a maioria das mães não encontra muitas dificuldades para que o filho as realize. No entanto, algumas inquietações foram relatadas, como observamos a seguir:

... O meu filho não tem um plano de exercícios, não caminha, não exerce nenhuma atividade física, mesmo tendo recebido todas as orientações..

... Durante o período que estava na natação o meu filho desenvolveu otite média serosa levando mais de um ano de acompanhamento sem comprometimento da acuidade auditiva. Gosta de atividade individual e intelectual como montar carrinho, jogos de pensar... Atualmente está praticando exercícios de bicicleta fazendo trilhas de 2 a $3 \mathrm{~h}$ por dia. Eu acho que é um exercício exagerado porque à noite tem apresentado hipoglicemia...

O exercício físico constitui-se em um dos pilares para o tratamento do diabetes mellitus associado à dieta e insulinoterapia, sendo referenciado pela literatura como uma forma de melhorar 0 grau de controle glicêmico e a auto-estima das pessoas diabéticas. No entanto, a atividade física deve ser acompanhada através da determinação de glicemia e glicosúria, evitando-se dessa maneira episódios de hipoglicemia. Mesmo conhecendo os benefícios que a atividade física proporciona, recomenda-se que a criança e adolescente considere a intensidade, duração, horário do exercício, estado do controle metabólico, tempo após a última refeição e o tempo de ação da insulina. Recomenda-se, ainda, que para iniciar um programa de exercícios, antes deva ser feita uma avaliação médica da criança e adolescente.

Os depoimentos das mães apontam que $8(26,7 \%)$ crianças e adolescentes sentem-se desmotivados para a prática de atividades físicas, o que nos leva a pensar que estes não estão informadas sobre as recomendações básicas e benefícios desta prática. Referem, ainda, que o filho apresenta episódios freqüentes de hipoglicemia. 0 desenvolvimento de tal quadro nestas circunstâncias nos conduz à hipótese de que o tipo de atividade eleita pode não estar adequada para o sexo, idade, peso, horário da última refeição, estado do controle metabólico e outros.

Acreditamos que um programa de educação em diabetes deva contemplar recomendações e orientações para a criança e os pais sobre atividades físicas, bem como os seus benefícios e possíveis 
intercorrências como os episódios de hipoglicemia. O mais importante é encorajá-los à adesão de atividade física tão logo quanto possível, pois só assim haverá uma possibilidade concreta da criança e do adolescente virem a assumir 0 auto-cuidado no futuro em relação ao diabetes, mantendo os níveis glicêmicos compatíveis com o controle metabólico.

Visualizamos que outra estratégia para estimular a criança à prática da atividade física poderia ser garantida ao introduzí-la nas escolas através de intercâmbios entre a equipe multidisciplinar de saúde e os professores de educação física da rede municipal e estadual de educação.

Analisando as dificuldades apresentadas pelas mães, no que diz respeito às relações do filho diabético com os demais membros da família, observamos que 25 mães (83,3\%) acreditam que o filho sente-se diferente dos demais irmãos, 22 (73,3\%) afirmam que eles usam a doença para conseguir algo a mais deles e 26 (86,7\%) referem que os problemas de ordem familiar interferem na obtenção de um bom controle metabólico do filho. Os depoimentos descritos a seguir mostram as diversas situações de dificuldades encontradas pelas mães:

... ela refere que é inútil, doente, porque não é normal, não presta para nada, para viver neste inferno tomando injeção todo dia, ficando roxa, que prefere morrer. Os irmãos acham que perdi o controle sobre ela, pois é mal-criada, responde perto de todo mundo, é revoltada e usa o diabetes para chantagear. A minha filha é nervosa, rebelde, irritada, portanto exige mais que os irmãos ...

... o meu filho sente-se diferente dos demais irmãos porque eu nunca o deixo sair por causa do diabetes. Ele usa sempre a doença para conseguir algo e na maioria das vezes consegue ...

Ao analisar o comportamento do filho diabético, referido pelas mães em relação aos demais membros da família, vimos que os achados confirmam aqueles encontrados por outros autores ${ }^{(2,7)}$.

Para compreender o comportamento da criança e do adolescente frente a uma doença crônica, os profissionais de saúde precisam estar informados a respeito dos estágios de desenvolvimento cognitivo e sócio-emocional destes, pois, a doença crônica afeta as interações da criança com o ambiente físico e social no qual vive. Nesta direção, a maneira e duração pela qual a doença crônica pode modificar os processos do desenvolvimento da pessoa dependem do tipo da doença, gravidade, história natural, prognóstico, grau de limitação, estrutura genética e implicações, necessidade de cuidado físico, aparência física, diferenças individuais no temperamento e personalidade, dinâmica interpessoal da família, rede de apoio social e financeiro, resposta dos professores, médicos, enfermeiras e outros profissionais ${ }^{(2)}$.

No entanto, precisamos estar atentas para o fato de que as implicações de uma doença crônica se dão a longo prazo, incidindo sobre desenvolvimento social, emocional e cognitivo, podendo diferir dependendo da individualidade de cada criança. As falas das mães sobre o comportamento da criança e do adolescente frente aos demais membros da família permitem-nos identificar a existência de mudanças na interação da criança com as mães e irmãos, interferindo nas relações familiares. Acreditamos também que as mães não têm apoio e suporte para enfrentar tais situações no dia-a-dia e, conseqüentemente, desenvolvem sentimentos de culpa e superproteção. Entendemos que a colaboração de um psicólogo dentro da equipe multiprofissional possa ajudá-las a lidar melhor com estas situações.

Quando indagamos como a mãe percebe o relacionamento da equipe profissional em relação ao controle do diabetes do filho obtivemos as respostas abaixo mencionadas:

... uma vez, tentei mudar de hospital para unidade básica de saúde, pois havia possibilidade de adquirir insulina gratuitamente ... Mas, senti muita diferença, passei a não ter confiança, não me senti segura ... O meu filho dizia: esta médica não entende nada ... Ele omitia informações à médica. Aguardava até as cinco horas da tarde para ser atendido, ficando exausto e nervoso, não respondendo adequadamente as perguntas da médica. $A$ médica sugeriu um acompanhamento psicológico para o meu filho e ele respondeu: eu não preciso de psiquiatra, eu não sou louco. A médica não levou em conta a crise familiar que estávamos passando naquele momento. Parece que os médicos não acreditam muito que os fatores externos interferem na glicemia. O médico sempre diz você tem que ter auto-controle ... Controle estas questões na sua casa ... Após este episódio voltou a realizar o tratamento no hospital o qual estava anteriormente..

... eu acho que o relacionamento com a equipe é muito rígido. Tinha medo o tempo todo da censura. Achava que ia levar uma "chamada" a cada retorno... Hoje com a mudança de médico está tudo bem...

... no começo as informações da equipe de saúde eram muito divergentes. Durante a primeira internação não sabia nada sobre diabetes e perguntei à enfermeira é grave? e ela respondeu: se você sabe rezar, reze... Fiquei com horror daquilo ... Quando a nutricionista veio fazer a orientação alimentar para irmos embora do hospital senti que ela tinha feito uma lavagem cerebral na minha cabeça. Ela dizia: faça omelete sem uma gota de óleo, tudo era para sempre, nunca mais ... Cheguei em casa com tanto medo, que não sabia nem fazer arroz mais ...

O conteúdo do depoimento das mães foi também analisado tendo em vista o relacionamento dessa clientela com a equipe de saúde. Este resultado mostrou problemas em várias esferas, desde a capacitação profissional para a organização dos serviços de saúde que prestam 0 atendimento requerido para este tipo de paciente. Reconhecemos que a própria estrutura do Sistema Único de Saúde no Brasil ainda não está totalmente implantada, havendo uma sobrecarga de atendimento no nível terciário o que pode estar comprometendo a qualidade do atendimento à criança e adolescente diabético. Por outro lado, a prestação dos serviços em saúde, na 
área hospitalar ou ambulatorial, está organizada para atender a doença, revelando-se uma forma de atendimento centrado na queixa e na conduta, sem preocupação com o seguimento e apoio contínuo ${ }^{(12)}$. Portanto, pensar em um programa de educação em diabetes para crianças e adolescentes passa obrigatoriamente pela organização dos serviços, priorizando a capacitação de profissionais, a fim de qualificar 0 atendimento a esta clientela, minimizar o impacto da doença na família e, minorar o sofrimento das crianças $e$ adolescentes e dos próprios pais em relação ao diabetes mellitus.

\section{CONSIDERAÇÕES FINAIS}

O conjunto de dados obtidos nesta investigação mostrounos que as mães estão envolvidas no cuidado diário com o filho e que elas enfrentam dificuldades para colaborar com o regime de tratamento e controle do diabetes. Tais dificuldades estão relacionadas à vários fatores: reestruturação do cardápio alimentar da família, motivação do filho à prática de exercícios físicos, adaptação escolar, relacionamento com os demais irmãos e relacionamento com a equipe de saúde. Essas situações de dificuldades indicam que as mães precisam de apoio e suporte para lidar com os filhos diabéticos.

Os dados encontrados nesta investigação merecem ser analisados pelos profissionais de saúde com vistas ao aprimoramento dos programas de assistência à criança e adolescente diabéticos. Neste sentido delimitamos algumas recomendações, as quais, apresentamos a seguir:

\section{REFERÊNCIAS BIBLIOGRÁFICAS}

1. Pond JS, Peters ML, Pannell DL, Rogers CS. Psychosocial challenges for children with insulin-dependent diabetes mellitus. Diabetes Educator 1995; 21(4):297-9.

2. Sabbeth B. Understanding the impact of chronic childhood illness on families. Pediatr Clin North Am 1984; 31(1):47-57.

3. Follansbee DS. Assuming responsibility for diabetes management: what age? what price? Diabetes Educ 1989; 15(4):347-53.

4. Saucier CP, Clark LM. The relationship between self-care and metabolic control in children with insulin-dependent diabetes mellitus. Diabetes Educ 1993; 19(2):133-5.

5. Kushion W, Salisbury PJ, Seitz KW, Wilson BE. Issues in the care of infants and toddlers with insulin - dependent diabetes mellitus. Diabetes Educ 1991; 17(2):107-10.

6. Warzak WJ, Majors CT, Ayllon T, Milan MA, Delcher HK. Parental versus profissional perceptions of pediatric diabetes care. Diabetes Educ 1993; 19(2):121-4.
- Formação e capacitação de equipes multiprofissionais especializadas para atender as crianças e adolescentes diabéticos tipo 1, o que exigirá esforços conjuntos entre instituições públicas e privadas.

- Intensificação de programas de educação em diabetes visando a redução de internações e prevenção das complicações advindas da doença, ou pelo menos, seu protelamento naqueles casos em que as complicações não puderem ser evitadas. Tratando-se de crianças e adolescentes que encontram-se na fase inicial da doença, esta ação deve ser prioritária.

- Fortalecimento e ampliação de grupos de educação em diabetes, que motivem as mães para uma participação efetiva, dando oportunidade de elaboração dos problemas enfrentados com os filhos. - Reconhecimento do enfermeiro, enquanto profissional da equipe de saúde, responsável pelo acompanhamento domiciliar das famílias com crianças e adolescentes diabéticos, comprometido com a educação e treinamento em relação aos aparelhos e recursos utilizados no domicílio.

- Desenvolvimento de eqüidade ao atendimento às famílias de crianças e adolescentes diabéticos tipo 1, através de apoio para que estas possam lidar com a criança portadora de doença crônica.

- Promoção de campanhas de esclarecimento junto à população através da imprensa escrita e falada, detecção precoce de diabetes, entre outras.

- Desenvolvimento de pesquisas no sentido de identificar os fatores que possam favorecer ou interferir no aprendizado das crianças e adolescentes para a auto-aplicação de insulina.

7. Murphy H. A diabetes na infância. Nurs Rev Técnica Enfermagem 1995; 8(92):20-3.

8. Pupo AA. Diabetes mellitus na criança e adolescente. In: Setian N, organizador. Endocrinologia pediátrica: aspectos físicos e metabólicos do recém-nascido ao adolescente. São Paulo(SP): Sarvier; 1989. p. 173-210.

9. Rull JA, Zorrilla E, Jadzinsky MN, Santiago JV. Diabetes Mellitus: complicações crônicas. México: Interamericana/Mc Graw-Hill; 1992. 10. Prochownek DC, Becker MH, Brow MB, Liang WM, Bennett S. Understanding young children's health beliefs and diabetes regimen adherence. Diabetes Educ 1993; 19(3):409-18.

11. Zanetti ML, Mendes IAC. Caracterização de crianças e adolescentes com diabetes mellitus tipo 1 em seguimento terapêutico. Rev Gauch Enfermagem 2000 janeiro: 21(1):82-99.

12. Zanetti ML, Mendes IAC, Ribeiro KP. O desafio para o controle domiciliar em crianças e adolescentes diabéticos tipo 1. Rev Latinoam Enfermagem 2001 julho; 9(4):32-6. 